\title{
Flexiblity of Using Com-Poisson Regression Model for Count Data
}

\author{
Esin AVCI * \\ Department of Statistics, Faculty of Arts and Sciences, Giresun University, Turkey
}

\begin{abstract}
The Poisson regression model is the most common model for fitting count data. However, it is suitable only for modeling equi-dispersed distribution. The Conway-Maxwell-Poisson (COM-Poisson) regression model allows modeling over and under-dispersion distribution. The purpose of this study is to demonstrate the flexibility of the Conway-MaxwellPoisson (COM-Poisson) regression model on simulation and alg data.
\end{abstract}

Keywords Count data,Poisson regression,COM-Poisson regression, under-dispersed count data, COMPoissonReg

AMS 2010 subject classifications 62P10,62-07

DOI: $10.19139 /$ soic.v6i2.278

\section{Introduction}

The number of occurrence of any event within a specified time can be described as counting data. In the case of the dependent variable is a count and researcher is interested in how this count changes as the explanatory variable increases count data regression model is used. Modelling count data has been widely used in actuarial sciences, Aitkin et al. (1990) [1] and Renshaw (1994) [19] fitted Poisson regression to two different set of U.K. motor claim data, and in biostatistics and demography,Frome (1983) [8] modelled the lung cancer death rates among British physicians who were regular cigarette smokers. In recent years this model has been used frequently in the economy, political science, and sociology, Lord (2006) [12] modeled motor vehicle crashes by using PoissonGamma model and Riphahn et al. (2003) [20] fitted the model to German Socioeconomic Panel (GSOEP) data. Because of counts are all positive integers and for rare events the Poisson distribution (rather than the normal) is appropriate. However, it is suitable only for modeling equi-dispersed (i.e., an equal mean and variance) distribution. Many real data do not adhere to this assumption (over- or under-dispersed data) and the inappropriate imposition of Poisson regression model may underestimate the standard errors and overstate the significance of regression coefficients. For over-dispersed data, the Generalized Estimation Equations (GEEs) method with negative Binomial distribution is a popular choice and increase the efficiency of estimates [12,9]. Other overdispersion models include Poisson mixtures [15] and quasi-Poisson model is characterized by the first two moments (mean and variance) [25]. However, these models are not suitable for under-dispersed data.Under-dispersed data is less commonly observed. In cases the sample is small and the sample mean is very low and can be caused by the data generating a process that is independent of the sample size or mean [18]. A few models exist that allow for both over- and underdispersed data. One example is the restricted generalized Poisson regression models of Famoye (1993)[6]. It is called restricted, because it belongs to an exponential family under the condition that the distribution parameter is constant. The Conway-Maxwell-Poisson(COM-Poisson)regression model is an alternative model to fit data sets of varying dispersion. The Conway-Maxwell-Poisson (COM-Poisson) distribution is a two-parameter generalization

$\overline{{ }^{*} \text { Correspondence }}$ to: Department of Statistics, Faculty of Arts and Sciences, Giresun University, Giresun, Turkey (28100). Email:esinavci@hotmail.com

ISSN 2310-5070 (online) ISSN 2311-004X (print)

Copyright (C) 2018 International Academic Press 
of the Poisson which also includes the Bernoulli and geometric distribution that allows for over- and underdispersion. The distribution was briefly introduced by Conway and Maxwell in 1962 for modeling queuing systems with state-dependent service rates. The statistical properties of the COM-Poisson distribution, as well as methods for estimating its parameters were established by Shmueli et al.(2005) [23]. The COM-Poisson distribution has been used in a variety of count data application, $[4,3,7,24,5,23,14,13,11]$.

\section{Methods}

\subsection{Poisson Regression Modes}

Poisson regression is a special case of Generalized Linear Models (GLM) framework. The simplest distribution used for modeling count data is the Poisson distribution. Denote by $\mathrm{Y}$ a random variable from the Poisson $U 3 b b$ distribution, with the distribution function given by

$$
P(Y=y \lambda)=\frac{\lambda^{y} e^{-\lambda}}{y !}, \quad \text { for } \quad y=0,1,2, \ldots
$$
$\hat{\beta}_{1}$

one of the properties of the Poisson distribution is that the ratio of consecutive probabilities is linear in y, or $\hat{\beta}_{0}$

$$
\frac{P(Y=y-1)}{P(Y=y)}=\frac{y}{\lambda}
$$

The canonical link is $g(\lambda)=\log (\lambda)$ resulting in a log-linear relationship between mean and linear predictor. The variance in the Poisson regression model is identical to the mean [27]. The mean Poisson regression model can be assumed to follow a log link, $E\left(Y_{i}\right)=\lambda_{i}=\exp \left(x_{i}^{\prime} \beta\right)$, where $x_{i}$ denotes the vector of explanatory variables and $U 3 b 2$ the vector of regression parameters. The maximum likelihood estimates can be obtained by maximizing the $\log$ likelihood.

\subsection{Negative Binomial Models}

The second way of modeling over-dispersed count data is to assume a Negative Binomial (NB) distribution for which can arise as a Gamma mixture of Poisson distributions. One parameterization of its probability density function is

$$
P(Y=y \backslash \lambda, \theta)=\frac{\Gamma(y+\theta)}{\Gamma(\theta) y !} \frac{\lambda^{y} \theta^{\theta}}{(\lambda+\theta)^{(y+\theta)}}, \quad \text { for } \quad y=0,1,2, \ldots
$$

with mean and shape parameter $\theta ; \Gamma($.$) is the Gamma function. For every fixed \theta$, this is another special case of the GLM framework. It also has $\phi=1$ but with variance function $V(\lambda)=\lambda+\frac{\lambda^{2}}{\theta}$ [27]. The mean of NB regression can also be assumed to follow the log link, $E\left(Y_{i}\right)=\lambda_{i}=\exp \left(x_{i}^{\prime} \beta\right)$, and the maximum likelihood estimates can be obtained by maximizing the log likelihood.

\subsection{Geometric Regression Model}

The geometric distribution is a generalized of Poisson distribution by including a gamma noise variable (Poissongamma mixture (negative Binomial)) which dispersion parameter is set to one. The probability distribution function of the geometric distribution is

$$
P(Y=y \backslash p)=p(1-p)^{y}, \quad \text { for } \quad y=0,1,2, \ldots
$$

In geometric regression model, the mean of $Y$ is determined by $E\left(Y_{i}\right)=\lambda_{i}=\exp \left(x_{i}^{\prime} \beta\right)$, where $x_{i}$ denotes the vector of explanatory variables and $U 3 b 2$ the vector of regression parameters. The regression coefficients are estimated using the method of maximum likelihood [2]. 


\subsection{COM-Poisson Regression Model}

The Conway-Maxwell-Poisson (COM-Poisson) is a generalization of the Poisson distribution which can model both under-dispersed and over-dispersed data. In some applications, the ratio in equation (2) may not decrease linearly in $y$, i.e.the distribution may have a thicker or thinner tail than the Poisson. Suppose instead of equation $(2)$,

$$
\frac{P(Y=y-1)}{P(Y=y)}=\frac{y^{v}}{\lambda}
$$

is set for a random variable $Y$. The resulting distribution for which equation (5) holds, called the ConwayMaxwell-Poisson distribution [26], is given by

$$
P(Y=y \backslash \lambda, v)=\frac{\lambda^{y}}{(y !)^{v}} \frac{1}{\left.\sum_{\substack{\lambda^{j} \\ j=0}}^{\infty}\right)^{v}}, \quad \text { for } \quad y=0,1,2, \ldots
$$

for $U 3 b b>0$ and $U 3 c 5 \geq 0$. This satisfies the conditions for a probability function. $v$ is considered the dispersion parameter such that $v \succ 1$ represents under-dispersion, and $v \prec 1$ over-dispersion.

Minka and et al. (2003) [16] denote the infinite sum in the denominator by:

$$
Z(\lambda, v)=\sum_{j=0}^{\infty} \frac{\lambda^{j}}{(j !) v}
$$

The COM-Poisson distribution includes three well-known distribution as special cases: Poisson $(v=$ $\left.1, Z(\lambda, v)=e^{\lambda}\right)$, Geometric $\left(v=0, \lambda \prec 1, Z(\lambda, v)=\frac{1}{1-\lambda}\right)$, and Bernoulli $\left(v \rightarrow \infty\right.$ with) probability $\left.\frac{\lambda}{1+\lambda}\right)$ [23].

Taking a GLM approach, Sellers and Shmueli (2010) [21] proposed a COM-Poisson regression model using the link function,

$$
\eta(E(Y))=\log \lambda=X^{\prime} \beta=\beta_{0}+\sum_{j=1}^{p} \beta_{j} X_{j}
$$

Accordingly, this function indirectly models the relationship between $E(Y)$ and $X^{\prime} \beta$, and allows for estimating $U 3 b 2$ and $v$ via associated normal equations. Because of the complexity of the normal equation, using $\beta^{(0)}$ and $v^{(0)}=1$, as starting values. These equations can thus be solved via an appropriate iterative reweighted least squares procedure (or by maximizing the liklihood function directly using an optimization program) to determine the maximum likelihood estimates $\hat{\beta}$, and $\hat{v}$. The associated standard errors of the estimated coefficients are derived using the Fisher Information matrix [22].

To comparison of different scale Poisson and COM-Poisson model coefficients, Sellers and Shmueli (2010) [21] offered to divide the COM-Poisson coefficients by $v$, because of $E\left(Y^{v}\right)=\lambda$.

\subsection{Testing for Variable Dispersion}

In GLM to detect over or under-dispersion simply, the researcher may look at the rule of thumb that the mean deviance, that is deviance/degree of freedom should be close to unity. Deviance theoretically allows one to determine if the fitted GLM model is significantly worse than the saturated model [17]. Sellers and Shmueli (2010) [21] established a hypothesis testing procedure to determine if significant data dispersion exists, thus demonstrating the need for a COM-Poisson regression model over a simple Poisson regression model; in other words, they test whether $(v=1)$ or otherwise [22]. The test statistics,

$$
C=-2 \log \Lambda=-2\left[\log L\left(\hat{\beta}^{(0)}, \hat{v}=1\right)-\log L(\hat{\beta}, \hat{v})\right]
$$

Where $\Lambda$ is the likelihood ratio test statistic, $\hat{\beta}^{(0)}$ are the maximum likelihood estimates obtained under $H_{0}: v=1$ (i.e., the Poisson estimates), and $(\hat{\beta}, \hat{v})$ are the maximum likelihood estimates under the general state space for the COM-Poisson distribution with 1 degree of freedom. For small samples, the test statistic distribution can be estimated via bootstarp [21]. 


\subsection{Akaike Information Criteria (AIC)}

When several models are available, one can compare the models performance based on several likelihood measures which have been proposed in the statistical literature. One of the most popular used measures is AIC. The AIC penalized a model with larger number of parameters and is defined as

$$
A I C=-2 \ln L+2 p
$$

where $\ln L$ denotes the fitted $\log$ likelihood and $p$ the number of parameters [10]. A relatively small value of AIC is favorable for the fitted model.

\section{Application}

\subsection{Simulated Data}

To demonstrate the flexibility of the COM-Poisson distribution, 500 data were derived from Poisson, geometric, negative Binom and COM-Poisson, respectively. The inversion method is particularly simple to sample an integer value from the COM-Poisson distribution. The COM-Poisson probabilities are summed up starting from $P(Y=0)$, until this sum exceeds the value of a simulated Uniform $(0,1)$ variable. $Y$ is then an observation from the COMPoisson distribution [16]. Goodness of fit (associated p-values provided in parentheses) of each distribution and estimated parameters were given in Table 1. The analysis are performed in R program. Respectievely, glm() function from "stats" package and cmp() function from "COMPoissonReg" package are used.

Table 1 illustrates that, while the Poisson and the geometric distribution are meaningful only for identical distribution, the negative Binom distribution was flexible for many distributions except under-dispersion. However, COM-Poisson distribution was flexible for all considered distributions. Furthermore the estimated parameters almost same for Poisson and negative Binomial distributions.

Table 1: Goodness of fit on simulated data of size 500

\begin{tabular}{|c|c|c|c|c|}
\hline \multirow[t]{2}{*}{ Distribution } & \multicolumn{4}{|c|}{ Estimated Parameter } \\
\hline & Poisson & Geometric & Negative Binom & COM-Poisson \\
\hline Poisson $(\lambda=5)$ & $\begin{array}{c}\lambda=5.064 \\
(0.366)\end{array}$ & $\begin{array}{l}p=0.165 \\
(0.000)\end{array}$ & $\begin{array}{c}\lambda=5.032, \\
\theta=188.897 \\
(0.757)\end{array}$ & $\begin{array}{c}\lambda=5.205, \\
v=1.030 \\
(0.787)\end{array}$ \\
\hline Geometric $(p=0.4)$ & $\begin{array}{c}\lambda=1.404 \\
(0.000)\end{array}$ & $\begin{array}{l}p=0.416 \\
(0.682)\end{array}$ & $\begin{array}{c}\lambda=1.404, \theta=0.953 \\
(0.600)\end{array}$ & $\begin{array}{c}\lambda=0.589, \\
v=0.007 \\
(0.780)\end{array}$ \\
\hline $\begin{array}{l}\text { Negative Binom } \\
\qquad(\lambda=6, \theta=10)\end{array}$ & $\begin{array}{c}\lambda=6.718 \\
(0.000)\end{array}$ & $\begin{array}{l}p=0.130 \\
(0.000)\end{array}$ & $\begin{array}{c}\lambda=6.834, \theta=10.484 \\
(0.762)\end{array}$ & $\begin{array}{c}\lambda=2.779, \\
v=0.550 \\
(0.911)\end{array}$ \\
\hline $\begin{array}{c}\text { COM-Poisson }(\lambda=15, \\
v=8)\end{array}$ & $\begin{array}{c}\lambda=0.988 \\
(0.000)\end{array}$ & $\begin{array}{l}p=0.503 \\
(0.000)\end{array}$ & $\begin{array}{c}\lambda=1.006, \theta=84618 \\
(0.000)\end{array}$ & $\begin{array}{c}\lambda=13.468, \\
v=8.030 \\
(0.166)\end{array}$ \\
\hline $\begin{array}{c}\text { COM-Poisson }(\lambda=4, \\
v=0.3)\end{array}$ & $\begin{array}{c}\lambda \\
=102.224 \\
(0.000)\end{array}$ & $\begin{array}{l}p=0.010 \\
(0.000)\end{array}$ & $\begin{array}{c}\lambda=102.222, \\
\theta=48.028 \\
(0.981)\end{array}$ & $\begin{array}{c}\lambda=3.6869, \\
v=0.283 \\
(0.911)\end{array}$ \\
\hline
\end{tabular}


To illustrate the flexibility of the COM-Poisson model, the considered distributions (Poisson, geometric, negative Binom and COM-Poisson) were above regressed with arbitrarily chosen single explanatory variable $X$ and determined coefficients $\left(\beta_{0}=2, \beta_{1}=0.2\right.$ ). By fitting the considered distribution (Poisson, geometric, negatif Binom and COM-Poisson) the estimated model parameters, Log likelihood and AIC values were given in Table 2.

Under the response variable has Poisson distribution, the Poisson regression model gave the smallest Loglikelihood and AIC values. Hence, the Poisson regression model shows the best fit among the estimated models for statistically significant coefficients. The second best fit model for statistically significant coefficients was the COM-Poisson regression model. The dispersion parameter $(v)$ was estimate quite near to 1 . Except the geometric regression model, the best fit was estimated with COM-Poisson regression model for geometric distributed responce. Also the dispersion parameter $(v)$ was estiamted 0. For negative Binomial distributed responce (overdispersed data), COM-Poisson and negative Binom were estimated the model parameters, Log likelihood and AIC values almost the same. The COM-Poisson regression model estimated the dispersion parameter $(v)$ as a 0.7496. Finally for COM-Poisson distributed responce, the Poisson and negative Binom regression models obtained similar estimation results. Although the geometric regression model gave the smallest Log likelihood and AIC values, the explanatory variable was not statistically significant. It was concluded from the Table 2, under different distributed responce variable the COM-Poisson regression model was the best model.

Table 2: Estimated model parameters and AIC for simulated data of size 500

\begin{tabular}{|c|c|c|c|c|}
\hline \multirow[t]{2}{*}{ Distribution } & \multicolumn{4}{|c|}{ Estimated Parameter } \\
\hline & Poisson & Geometric & Negative Binom & COM-Poisson \\
\hline \multirow{5}{*}{$\begin{array}{l}\text { Poisson } \\
\left(\boldsymbol{\beta}_{0}=2, \boldsymbol{\beta}_{1}=0.2\right)\end{array}$} & $\hat{\beta}_{0}=2.0156(0.0331)^{*}$ & $\hat{\beta}_{0}=-2.0172(0.0986)^{*}$ & $\hat{\beta}_{0}=2.0157(0.0340)^{*}$ & $\hat{\beta}_{0}=1.8627(0.1325)^{*}$ \\
\hline & $\hat{\beta}_{1}=0.2194(0.0553)^{*}$ & $\hat{\beta}_{1}=-0.2164(0.1685)$ & $\hat{\beta}_{1}=0.2191(0.0570)^{*}$ & $\hat{\beta}_{1}=0.2043(0.0538)^{*}$ \\
\hline & Loglik $=-1254.05$ & Loglik $=-500.621$ & Loglik $=-1253.583$ & Loglik $=-1253.375$ \\
\hline & $\mathrm{AIC}=2512.1$ & $\mathrm{AIC}=1005.2$ & $\mathrm{AIC}=2513.2$ & $\mathrm{AIC}=2512.75$ \\
\hline & & & $\hat{\theta}=133$ & $v=0.9266$ \\
\hline \multirow{5}{*}{$\begin{array}{l}\text { Geometric } \\
\left(\boldsymbol{\beta}_{0}=2, \boldsymbol{\beta}_{1}=0.2,\right. \\
\theta=1)\end{array}$} & $\hat{\beta}_{0}=1.8778(0.0342)^{*}$ & $\hat{\beta}_{0}=1.8830(0.0988)^{*}$ & $\hat{\beta}_{0}=1.8830(0.1008)^{*}$ & $\hat{\beta}_{0}=0.6458(0.0542)^{*}$ \\
\hline & $\hat{\beta}_{1}=0.4653(0.0557)^{*}$ & $\hat{\beta}_{1}=0.4555(0.1683)^{*}$ & $\hat{\beta}_{1}=0.4555(0.1721)^{*}$ & $\hat{\beta}_{1}=0.1449(0.0567)^{*}$ \\
\hline & Loglik=-2847.861 & Loglik $=-691.485$ & Loglik $=-1587.09$ & Loglik $=-1010.137$ \\
\hline & $\mathrm{AIC}=5699.7$ & $\mathrm{AIC}=1387$ & $\mathrm{AIC}=3180.2$ & $\mathrm{AIC}=2026.274$ \\
\hline & & & $\hat{\theta}=0.9710$ & $v=0.000$ \\
\hline \multirow{5}{*}{$\begin{array}{l}\text { Negative } \\
\text { Binom } \\
\left(\boldsymbol{\beta}_{0}=2, \boldsymbol{\beta}_{1}=0.2 \text {, }\right. \\
\theta=10)\end{array}$} & $\hat{\beta}_{0}=1.9205(0.0623)^{*}$ & $\hat{\beta}_{0}=0.5198(0.1079)^{*}$ & $\hat{\beta}_{0}=1.9214(0.0684)^{*}$ & $\hat{\beta}_{0}=1.9205(0.0926)^{*}$ \\
\hline & $\hat{\beta}_{1}=0.2281(0.1028)^{*}$ & $\hat{\beta}_{1}=0.2247(0.1811)$ & $\hat{\beta}_{1}=0.2273(0.1133)^{*}$ & $\hat{\beta}_{1}=0.1881(0.0941)^{*}$ \\
\hline & Loglik=-913.069 & Loglik $=-481.29$ & Loglik $=-908.422$ & Loglik=-907.92 \\
\hline & $\mathrm{AIC}=1830.1$ & $\mathrm{AIC}=966.58$ & $\mathrm{AIC}=1822.8$ & $\mathrm{AIC}=1821.84$ \\
\hline & & & $\hat{\theta}=10.03$ & $v=0.7496$ \\
\hline \multirow{5}{*}{$\begin{array}{l}\text { COM-Poisson } \\
\left(\beta_{0}=2, \beta_{1}=0.2,\right. \\
\lambda=15, v=8)\end{array}$} & $\hat{\beta}_{0}=-0.0813(0.0930)$ & $\hat{\beta}_{0}=0.0813(0.1291)$ & $\hat{\beta}_{0}=-0.0813(0.0930)$ & $\hat{\beta}_{0}=2.1119(0.2660)^{*}$ \\
\hline & $\hat{\beta}_{1}=0.0423(0.1581)$ & $\hat{\beta}_{1}=-0.0423(0.2202)$ & $\hat{\beta}_{1}=0.0423(0.1581)$ & $\hat{\beta}_{1}=0.3364(0.0446)^{*}$ \\
\hline & Loglik $=-510.2$ & Loglik $=-350.725$ & Loglik $=-510.199$ & Loglik $=-220.285$ \\
\hline & $\mathrm{AIC}=1024.4$ & $\mathrm{AIC}=705$ & $\mathrm{AIC}=1026.4$ & $\mathrm{AIC}=446.57$ \\
\hline & & & $\hat{\theta}=130961$ & $v=8.084$ \\
\hline
\end{tabular}

* 5\% statistically significant 


\subsection{Cocconeis Placentula Ehrenberg Data}

Cocconeis Placentula Ehrenbergis one of the Epilithic algae occur in Freshwater (more oligotrophic) habitats, including slightly humic waters. Between June 2013 and May 2014, the number of Cocconeis Placentula Ehrenbergdata are collected from four different stations that located on Batlama stream. The descriptive statistics was given in Table 3.

Table 3. Descriptive statistics

\begin{tabular}{cccc}
\hline & & Median & $\begin{array}{c}\text { Range } \\
\text { (Max-Min) }\end{array}$ \\
\hline All & & 8 & $(3-50)$ \\
Season & Summer & 0.5 & $(4-11)$ \\
& Autumn & 1 & $(3-7)$ \\
& Winter & 21.5 & $(11-38)$ \\
& Spring & 32 & $(15-50)$ \\
& & & \\
& I & 9 & $(26-35)$ \\
& II & 8.5 & $(35-50)$ \\
& III & 1.5 & $(20-35)$ \\
& IV & 3 & $(25-38)$ \\
\hline
\end{tabular}

In order to model the effect of stations and season on the number of Cocconeis Placentula Ehrenberg the models described above applied to the data set. At the end of the section, all fitted models are compared highlighting that the modeled mean function is similar but the fitted likelihood and AIC are different.

Results of regressing the number of Cocconeis Placentula Ehrenberg on stations and season for Poisson and COM-Poisson models are given in Table 4. Table 4 shows that the parameter estimates, standard error and AIC value. After dividing the COM-Poisson coefficients by dispersion parameter $(v)$, the results in Table 4 indicate that the regression parameters for all models have similar estimates in terms of the coefficient magnitudes.

Table 4. Parameter estimates, standard error and AIC value for models

\begin{tabular}{|c|c|c|c|c|c|}
\hline & & \multicolumn{2}{|c|}{ Poisson } & \multicolumn{2}{|c|}{ COM-Poisson } \\
\hline & & $\begin{array}{c}\text { Estimated } \\
\text { Coefficient }\end{array}$ & $\begin{array}{l}\text { Standard } \\
\text { Error }\end{array}$ & $\begin{array}{c}\text { Estimated } \\
\text { Coefficient }\end{array}$ & Standard Error \\
\hline Intercept & & 1.8820 & $0.2143^{*}$ & $1.9246^{*}$ & $0.9123^{*}$ \\
\hline Season & $\begin{array}{l}\text { Summer } \\
\text { Autumn } \\
\text { Winter } \\
\text { Spring }\end{array}$ & $\begin{array}{c}- \\
-0.2336 \\
2.1832 \\
2.7327\end{array}$ & $\begin{array}{c}- \\
0.3071 \\
0.2153^{*} \\
0.2107^{*}\end{array}$ & $\begin{array}{c}- \\
-0.2299 \\
2.1745^{*} \\
3.1261^{*}\end{array}$ & $\begin{array}{c}- \\
0.3436 \\
0.9597 * \\
1.3285^{*}\end{array}$ \\
\hline Station & $\begin{array}{l}\text { I } \\
\text { II } \\
\text { III } \\
\text { IV }\end{array}$ & $\begin{array}{c}- \\
0.1567 \\
-0.4143 \\
-0.1929\end{array}$ & $\begin{array}{c}- \\
0.1042 \\
0.1212 * \\
0.1138 *\end{array}$ & $\begin{array}{c}- \\
0.3353 \\
-0.7015^{*} \\
-0.4239\end{array}$ & $\begin{array}{c}- \\
0.2209 \\
0.3280^{*} \\
0.2554\end{array}$ \\
\hline $\begin{array}{l}\text { Dispersion } \\
\text { parameter }\end{array}$ & & 0.3015 & - & 1.1790 & {$[95 \% \mathrm{CI}=(1.13 ; 2.89)]$} \\
\hline & $\begin{array}{c}\text { Log- } \\
\text { liklihood } \\
\text { AIC }\end{array}$ & -5 & & & $\begin{array}{l}-3.91 \\
9.42\end{array}$ \\
\hline
\end{tabular}

While the estimated dispersion parameter for Poisson regression model is 0.3015 , for COM-Poisson model is $v=1.1790$, indicating under-dispersion. To determine whether the dispersion parameter is significiant or not a 
hypothesis test which established by Sellers and Shmueli (2010) [21] was used. The p value was found 0.0000, and the 95\% bootstrap confidence interval for $v$ not include the value 1 (using 1000 samples). Indicating dispersion that requires a COM-Poisson regression instead of Poisson regression.

While the same season (Winter and Spring) was statistically significant for both regression model, the fourth station was statistically significant only for the Poisson regression model. In terms of Log-likelihood and AIC, the COM-Poisson regression showed best fit for data.

In terms of model interpretation, dividing the COM-Poisson coefficients by $v$ and taking exponent of each divided parameters, the COM-Poisson regression indicates that, according to summer referance category, respectively Winter and Spring are about 9 and 23 times as common to create Cocconeis Placentula Ehrenberg. Cocconeis Placentula Ehrenberg is about twice as common in first station than in third station.

\section{4. conclusion}

This study is related to the response variable of interest is a count, that is, takes on non-negative integer values. For count data, the most widely used regression model is a Poisson regression. Poisson regression is limiting in equidispersion assumption. Lately when the data display over- and under-dispersion COM-Poisson regression is used. To demonstrate the flexibility of the COM-Poisson distribution, 500 data were derived from Poisson, geometric, negative Binom and COM-Poisson, respectively. The goodness of fit test showed that, while the Poisson and the geometric distribution are meaningful only for identical distribution, the negative binom distribution was flexible for many distributions except under-dispersion. However, COM-Poisson distribution was flexible for all considered distributions. In terms of the model, the COM-Poisson regression model showed best fit for data based on Log-likelihood and AIC values. In order to model the effect of stations and season on the number of Cocconeis Placentula Ehrenberg, Poisson and COM-Poisson regression models are fitted respectively. The results indicated that the regression parameters of all models had similar estimates. According to the test which established by Sellers and Shmueli (2010) [21] to test whether the dispersion parameter is significant or not, indicated that COMPoisson regression was more adequate than Poisson model. It revealed that, while the same season (Winter and Spring) was statistically significant for both regression model, the fourth station was statistically significant only for the Poisson regression model. In terms of Log-likelihood and AIC, the COM-Poisson shows best fit for data. In terms of model interpretation, according to summer reference category, respectively Winter and Spring are about 9 and 23 times as common to create Cocconeis Placentula Ehrenberg. Cocconeis Placentula Ehrenberg is about twice as common in the first station than in the third station.

\section{Acknowledgement}

The author thank Kimberly Sellers for her insightful comments and help to run COMPoissonReg code.

\section{REFERENCES}

1. Aitkie, M., Anderson, D., Francis, B., Hinde, J., Statistical modeling in GLIM. New York: Oxford University Press, (1990).

2. Cameron, A.C., Trivedi, P.K., Regression analysis of countdata, New York: Cambridge University Press, 53, (2013).

3. Consul, P.C., Famoye, F.,Generalized Poisson regression model, Communications in Statistics (Theory \& Method), 2(1): 89109,(1992).

4. Consul, P.C., Generalized Poisson Distribution: Properties and Application. New York: Marcel Dekker (1989).

5. Conway, R.W., Maxweal, W.L.,A queuing model with state dependent service rates, Journal of Industrial Engineering, 12:132$136,(1962)$.

6. Famoye, F., Restricted generalized Poissonregression models, Commun.Statist.Theor. Meth,22:1335-1354, (1993).

7. Famope, F., Wulu, J.o., Singh, K.P.,On the generalized Poisson regression model with an application to accident data, Journal of Data Science, 2: 287-295, (2004).

8. Frime, E.L., The analysis of rates using Poisson regression models , Biometrics, 39: 665-674, (1983).

9. Hidbe, J.M., Negative Binomialregressio, Second edition, Cambridge University Press,(2011). 
10. Ismail, N., Zamani, H., Estimation of Claim Count Data using Negative Binomial, Generalized Poisson, Zero-Inflated Negative Binomial and Zero-Inflated Generalized Poisson Regression Models, Casualty Actuarial Society E-Forum(Spring 2013).

11. Khan, N.M. and Khan, M.H., Model for analysing count with over-, equi-and under-dispersion in actuarial statistics, Journal of Mathematics and Statistics, 6(2):92-95, (2010).

12. Lord, D., Modelling motor vehicle crashesusing Poisnon-Gamma models: Examining the effects of low sample mean values and small sample size on the estimation of the fixed dispersion parameter, Accident Analysis\&Prevention, 38(4): 751-766, (2006).

13. Lord, D., Geedipally, S.R., Guikema, S.D.,Extension of the application of Conway-Maxwell-Poisson models: analyzing traffic crash data exhibiting under-dispersion, Risk Analysis, 30(8): 1268-1276, (2010).

14. Lord, D., Guikema, S.D., Geedipally, S.R.,Application of the Conway-Maxwell-Poisson generalized linear model for analyzing motor vehicle crashes, Accident Analysns \& Preventioi, 40(3): 1123-1134, (2008).

15. McLachlan, G.J., On the EM algorithm for overdispersed count data, Statistical methods in Medical Research, 6: 76-98, (1997).

16. Minka, T.P., Shmueli, G., Kadane, J.B., Borle, S., Boatwright, P., Computing with the COM-Poisson distribution, Technical Report Series, Carnegie Mellon University Department of Statistics, Pennsylvania, (2003).

17. Myers, R.H., Montgomery, D.C., Vining, G.G., Generalized Linear Models with Applications in Engineering and the Science, John Wiley \& Sons INC,(2001).

18. Oh, J., Washington, S.P., Nam, D., Accident prediction model cor railway-highway interfaces, Accident Analysis\&Prevention, 38(2): 346-356, (2006).

19. Renshaw, A. E.,Modelling the claims process in the presence of covariates, ASTIN Bulletin, 24(2): 265-285, (1994).

20. Riphahn, R., Wambach, A., Million, A., Incentive effect in the demand for health care: a bivariate panel count data estimation, Journal of Applied Econometrics, 18(4): 387-405, (2003).

21. Sellers, K.F., Shmueli, G.,A Flexible Regression Model for Count Data, The Annals of Applied Statletics, 4(2): 943-961, (2010).

22. Sellers, K.F.,Shmueli, G.,Data Dispersion: Now you see it...Now you don't, Communication in Statistics: Theory and Methods, 42(17):3134-47, (2013)

23. Shmueli, G., Minka, T.P., Kadane, J.B., Borle, S., Boatwright, P.,A Useful Distribution for Fitting Discrete Data: Revival of the Conway-Maxwell-Poisson Distribution, Journal of The Royal Statistical Society. Series C (Applied Statistics), 54(1): 127-142, (2005).

24. Wang, W., Famoye, F.,Modeling household fertility decisions with generalized Poisson regression,Journay of Population Economics, 10: 273-283, (1997).

25. Wedderburn, R.W.M., Quasi-likelihood functions, generalized linear models, and the Gauss-Newton method, Biometrika, 61: 439447, (1974).

26. Wimmer, G. and Altmann, G., Thesaurus of univariate discrete probability distributions, Essen: Stamn. www.stamn.de/thesaurus/, (1999).

27. Zeileis, A., Kleiber, C., Jaskman, S.,Regression Models for Count Data in R, Journal of Statistical Software, 27(8): 1-25, (2008). 